\title{
Variations
}

Variations

Revue internationale de théorie critique

8 | 2006

Subjectivités libres et critique de la répression

\section{La fragilité du « Grand Frère »}

Lire 1984 et se souvenir

\section{Lucia Sagradini}

\section{(2) OpenEdition}

1 Journals

Édition électronique

URL : http://journals.openedition.org/variations/502

DOI : $10.4000 /$ variations. 502

ISSN : 1968-3960

Éditeur

Les amis de Variations

Édition imprimée

Date de publication : 1 novembre 2006

Pagination : 40-49

Référence électronique

Lucia Sagradini, «La fragilité du « Grand Frère » », Variations [En ligne], 8 | 2006, mis en ligne le 27 décembre 2012, consulté le 05 mai 2019. URL : http://journals.openedition.org/variations/502 ; DOI : 10.4000/variations.502

Ce document a été généré automatiquement le 5 mai 2019.

Les ami•e•s de Variations 


\title{
La fragilité du « Grand Frère »
}

\author{
Lire 1984 et se souvenir
}

\section{Lucia Sagradini}

1 Alors que le discours répressif devient omniprésent dans l'espace public, en même temps que surgissent les résistances et les révoltes à l'autoritarisme, de plus en plus nombreuses, je propose de faire retour sur George Orwell ${ }^{1}$. Lire ou relire 1984 signifie retracer le parcours de Winston Smith, protagoniste du roman. Il s'agit de souligner combien son expérience de vie, les souvenirs qui lui reviennent en mémoire forment un noyau dur à partir duquel il va construire son désir de révolte et de liberté. Cette orientation cherche à indiquer les entrecroisements entre subjectivité (sphère intime) et émancipation politique (sphère publique).

2 Marx dit dans Le 18 Brumaire de Louis Bonaparte : «Le poids des générations passées pèse encore, tel un cauchemar, sur le cerveau des vivants ${ }^{2} »$. Il est difficile d'ignorer cette capacité à nous hanter que possède le passé sur le présent. Marx indique la force du passé venant arrêter et suspendre le geste politique. Les individus pris dans la révolte, mais aussi dans la peur des possibilités qui pourraient s'ouvrir à eux, se retiennent et s'arrêtent. Le passé est alors ce qui vient empêcher le franchissement et l'expérience présente du dépassement.

Les travaux de Freud ont poursuivi cette idée du poids du passé, mais en montrant l'impact du vécu et des premières années sur la subjectivité et la vie des êtres vivants. Dans ce cadre particulier de la psychanalyse, il a même été soutenu que tout se jouait avant 4 ans. Aujourd'hui, les décisions gouvernementales pervertissent cette idée en proposant un contrôle et un encadrement oppressif des petits enfants, montrant des "signes de risque ", et en décidant de créer des suspects en bas âge ${ }^{3}$. Ainsi, le monde dans lequel nous nous trouvons et que nous tentons de dessiner par l'action politique est esquissé et marqué par avance, tout comme la vie intime et la subjectivité des êtres ; il est surtout entravé par le passé qui vient, avec sa horde de fantômes et de cauchemars, écraser l'action et la pensée des êtres.

4 Cependant, tout comme dans la psychanalyse, où un geste d'amour est considéré fondateur pour l'individu, la lecture de Walter Benjamin nous entraîne à envisager le passé dans sa possibilité réelle d'être porteur de promesse. Il y a chez Benjamin une 
inversion de la problématique de Marx, inversion qui considère le passé comme souffle de liberté que les individus et le présent peuvent saisir à tout moment, afin de réactualiser les expériences antérieures de liberté. Ainsi, les années non seulement pèsent sur les individus, mais peuvent aussi les construire et leur livrer les secrets du dépassement.

\section{Journal intime}

5 C'est pourquoi nous avons choisi de saisir la situation de Winston Smith, personnage principal de 1984, en nous concentrant sur une étude de son langage et de ses souvenirs, pour mettre à jour le parcours qui le mène à la rupture, non sans avoir été interpellés par un article de Claude Lefort ${ }^{4}$. Dans "Le corps interposé », l'auteur nous donne une clé de lecture pour comprendre la trame invisible qui amène les deux personnages principaux à ne plus être en lien: l'importance imprévisible de la trahison, en donnant le corps de l'autre, lors de la scène de torture et ce afin de préserver leur propre sauvegarde. Cependant, nous faisons un cheminement contraire à Lefort, en cherchant à faire surgir non pas l'expérience de peur lointaine et archaïque sur laquelle se fonde la torture du protagoniste, mais bien plutôt en explorant les expressions d'expérience de la liberté venues du passé et qui se tiennent dans les lignes du roman. Expériences qui vont lui permettre de désirer un autre monde, une autre vie. Notre choix de faire retour sur 1984 est dû à la singularité et la richesse de l'ouvrage sur la question de la subjectivité et de l'expérience comme prémices du dépassement. Avant tout, il est important de rappeler les conditions dans lesquelles se trouve Winston Smith. Il vit dans un cosmos organisé et rythmé par le Parti et sous l'effigie de Big Brother, l'incarnation de la soumission de tous à la puissance de l'un. Cadre moyen du Parti, il vit seul, sans avoir la possibilité de créer des liens avec les autres hommes, et toujours sous la surveillance d'un télécran. Son travail consiste à réécrire les articles de presse afin que le Parti ne connaisse pas la contradiction. La nourriture comme les mots ou les contacts sont rationnés, dosés par le Parti. Les individus sont ainsi maintenus « sous régime » et toute singularité de leur part les conduit à être vaporisés. Non seulement ils sont éliminés, mais les traces de leur existence sont effacées, car le Parti ne supporte aucune contestation, ni passée ni présente. Pourtant, Winston Smith va faire un acte de rébellion en décidant d'écrire un journal intime qui va, surtout, l'entraîner à repenser le régime sous lequel il vit ${ }^{5}$. Car, tenir un journal intime c'est choisir de réintroduire un dialogue avec soi-même. Le journal intime s'offre alors comme le support à l'essor d'une pensée et aussi à l'autonomie de la pensée : il sort l'individu du monde dans lequel il est et lui permet de construire les conditions de la réflexion par la mise à distance du monde et le retour sur soi. Le journal intime est une façon de sortir l'individu de la masse, puisque ce dialogue à soi produit de la pensée (autonomie); il est contraire au régime et situe Winston dans la transgression. Ainsi, l'écriture du journal intime est le moyen pour Winston de renouer avec un univers interne, de retrouver ses propres émotions et, dans cette remise en lien avec sa subjectivité, de se retrouver lui-même. En trouvant un moyen de se réinscrire comme être au monde, Winston plante la question de sa mise à distance de la masse et du régime.

6 Orwell réalise cette transposition de mise en écriture de l'expérience d'une subjectivité particulière dans l'expérience d'une société totalitaire. Là où Hannah Arendt va réaliser une conceptualisation du système totalitaire qui souligne la manière dont il détruit les liens verticaux - entre les générations - et horizontaux - entre les individus -, théorisant cette particularité de l'individu dans le système totalitaire de « coller » au mouvement du 
totalitarisme qui produit une fiction et vit dans le mensonge ${ }^{6}$, Orwell, lui, parvient à trouver une expression romancée de cette description analytique du totalitarisme. La liberté du roman est de permettre à Orwell d'inventer un parcours singulier, illustrant le détachement progressif et la révolte à cette situation de fiction. Winston, en décidant de reprendre une pensée intime et d'ouvrir le dialogue à soi-même, va ainsi pouvoir sortir de la fiction du système totalitaire. La place de la subjectivité et des expériences de l'intime se constitue, en conséquence, comme premier geste vers la révolte et la résistance au système.

7 Il est important de remarquer dans la tradition « distopique » (des romans mettant en scène un monde de domination et d'oppression) la différence entre Huxley et Orwell ${ }^{7}$. Si Orwell construit un univers où les formes de domination et d'oppression cherchent à isoler et contrôler l'individu, il reste que dans 1984 l'individu sait que la société dans laquelle il vit est inique. Dans Le Meilleur des mondes, l'usage combiné de la drogue et du divertissement, associé à la technique de clonage des êtres, parvient à tenir les individus dans l'illusion d'être dans «un monde merveilleux » en les coupant par la chimie et la technique de la réalité vécue, en les éloignant de leurs propres ressentis. Ainsi, l'angle d'attaque apporté par Huxley réaffirme par l'usage donné à la technique et aux expédients chimiques que l'action des systèmes répressifs est aussi orientée par une action de transformation et de séparation de l'individu avec sa propre subjectivité, espace intime où percevoir la réalité et sa véracité. Cette ligne de fictions se poursuit avec le film Matrix, où le personnage de Neo va lui aussi devoir faire l'effort de s'extraire d'un univers fictionnel qui le tient dans une profonde léthargie. Cette séparation de l'être à soi est aussi accentuée par la disparition de l'origine de l'individu: la venue au monde n'est plus le lieu d'une histoire et d'une rencontre amoureuse, mais le fait de la technique. Il y a aujourd'hui dans ces fictions d'anticipation le renforcement de l'oppression par la coupure radicale de l'être à sa propre existence, qui rend le détachement de la fiction toujours plus difficultueux, jusqu'à se perdre dans la ligne de fuite d'une émancipation impossible.

\section{Rêves d'amour}

8 Aujourd'hui encore, comprendre comment Winston arrive à cette rupture, au rejet d'un ordre établi auquel il participe activement, est une manière de saisir les multiples pistes de réflexion ouvertes par Orwell sur le système totalitaire et sur la question de la subjectivité. Tout d'abord, Orwell met en scène une particularité de son personnage. Winston rêve beaucoup et, de ses rêves, il réussit à réécrire une histoire, son histoire : les rêves lui permettent de faire resurgir de sa mémoire que, contrairement à ce qu'il croyait, il n'a pas tué sa mère. C'est un rêve de sa mère et de sa soeur sur un bateau qui coule, tandis que lui s'élève, qui va précéder et entraîner l'achat d'un cahier, qui deviendra le journal de Winston. Les rêves ont pour effet de le re-lier au passé et de lui permettre par la suite de penser qu'une façon différente de vivre est possible, puisque, avant, il y avait autre chose que le Parti et la Novlangue, la langue officielle ${ }^{8}$. Orwell construit l'expérience de la résistance de son protagoniste en réalisant un contre Nietzsche tout à fait saisissant. Si Nietzsche dans La Généalogie de la morale inscrit la mémoire du côté du dressage social de l'individu, moyen de parvenir à la soumission de l'individu à la collectivité et à ses règles, Orwell ouvre la problématique d'une mémoire porteuse d'émancipation'. Dans 1984, il nous indique ainsi le chemin d'une réflexion sur les formes de mémoire. Si d'un 
côté, la mémoire est l'instrument du dressage de l'individu, Nietzsche signale que cette mémoire se réalise par l'usage de la violence et les sévices corporels; de l'autre, Orwell nous parle d'une mémoire surgie des souvenirs d'expériences d'amour. Nietzsche explore la question de la mémoire en l'inscrivant du côté des fers rouges, la mémoire est alors marquage des corps. Orwell, lui, évoque une mémoire faite de gestes, qu'un coeur donne à un autre. Il nous offre alors une pensée profonde et intime: celle de mettre en lien le geste enveloppant à l'expérience de la liberté. Pour Orwell, l'amour est un geste qui participe à la construction de la liberté. Ainsi, la mémoire, trouvant comme expression des souvenirs heureux, ne participe et ne peut participer à un monde faux et fictionnel, construit sur la soumission, la peur et la haine. D'ailleurs, la mémoire qu'énonce Orwell est une mémoire flottante, car elle se tient repliée dans les zones brumeuses de l'inconscient du personnage et surgit de manière inattendue, lorsque Winston assiste à la projection d'un film de propagande, bien nommé Les Trente secondes de la haine. C'est-àdire que cette mémoire est aussi une mémoire qui s'oublie, un souvenir, quelque chose qui peut aller et venir au gré des expériences de l'individu. Alors, une image censée être porteuse de l'idéologie de Big Brother est déviée de ce pourquoi elle a été projetée. L'esprit de Winston réalise une association inattendue qui sort de la volonté autoritaire du régime : il y puise une activation inconsciente d'un souvenir oublié, mais présent ${ }^{10}$. Pour Orwell, le souvenir est alors le moyen pour l'individu de refaire l'expérience de soi et ainsi de sa propre différence. Ce faisant, le souvenir est porteur de résistances car il permet de penser l'hétéronomie de soi, de l'autre et du monde.

9 Ainsi, les rêves rappellent à Winston qu'enfant, il avait une mère qui les aimait, sa petite soeur et lui. Cette expérience disparue avec la façon dont sa mère a été vaporisée est au coeur des raisons qui le conduisent à s'interroger, et à rompre avec le régime. Il faut souligner que cette expérience personnelle peut refaire surface à la conscience de Winston grâce à une image du film de propagande. Winston est frappé par cette image où l'on voit, sur une barque, une mère juive protéger son enfant de son bras avant de mourir sous un bombardement. C'est cette image qu'il va retranscrire en premier lieu dans son carnet. Cette image aura donc sur Winston un effet imprévisible pour le Parti : celui de lui permettre de retrouver le fil perdu, coupé par le régime, de son histoire. Une association que le Parti n'a jamais pu envisager, et qui indique la nature de l'image : se donner à l'association libre de l'individu.

10 Pour Winston, retrouver son histoire c'est comprendre que le passé était différent ; et ce constat lui permet de penser qu'il vit dans une fiction mensongère construite par le Parti et qu'elle s'éloigne de la vérité et même de la réalité (voir le chapitre « Deux et deux font quatre »). Ainsi Winston, par la redécouverte de son passé, peut prendre conscience qu'il vit à l'intérieur d'une fiction et tente de s'en dégager. Sa rupture, si elle vient donc d'un retour en arrière, montre que les premiers pas à faire sont de réussir à comprendre pour l'individu où il est et ce qu'il vit. Primordial travail vers la liberté.

11 La gestuelle d'une femme dans le film de propagande, son geste enveloppant et protecteur est non seulement un détonateur, qui conduit Winston à faire des rêves de plus en plus précis sur sa mère, mais elle marque aussi sa fascination pour la manière dont les personnes s'expriment corporellement. Tout au long de l'ouvrage, il s'attache aux gestes des autres. Ces gestes sont sans doute l'expression indirecte d'expériences incorporées échappant au système et au langage.

12 Ainsi, les rêves porteurs de souvenirs lui permettent d'éveiller sa conscience et sont en fait les premiers pas vers le réveil, puisqu'ils font surgir la discordance avec le régime, 
inscrite dans l'expérience personnelle de Winston. Non seulement l'amour est une chose possible puisqu'il a existé, mais il peut exister à nouveau. Mais les rêves font aussi surgir la vérité de son rapport à sa mère : il y avait de l'amour entre eux et, surtout, il n'est pas responsable de sa mort. Ainsi, le régime totalitaire se construit sur la désolation et l'isolement des êtres qui ne peuvent plus construire de liens ni au monde ni à l'autre, et ne serait-ce qu'à eux-mêmes. La traversée de Winston Smith est aussi la traversée du désert politique et social dans lequel il erre, évoquant la situation réelle d'Orwell ${ }^{11}$. Les rêves, le journal intime, la rencontre avec Julia, une jeune femme que Winston va aimer, tous ces éléments concourent à nous indiquer le chemin pris pour sortir de la désolation.

\section{Les entrelacs dialectiques de la subjectivité et de l'émancipation}

Orwell insiste encore sur le goût de Winston Smith pour le passé. Il est exprimé dans son désir de posséder des objets d'un autre temps: ainsi le livre est valorisé par son ancienneté ; le presse-papier, qui a en son centre un morceau de corail, renferme une époque lointaine et porteuse de mystères. Par leur ancienneté et ce qu'ils sous-entendent d'un « avant ", les objets sont donc les supports latents sur lesquels Winston va projeter ses désirs, ses rêves. Son goût pour le passé se manifeste aussi par son attraction pour les personnes ayant vécu avant la révolution, par exemple sa rencontre avec «le vieux prolétaire ", ou la confiance aveugle qu'il porte à l'antiquaire qui lui loue une chambre « comme dans le temps ». Ou même à O'Brien, membre du Parti Intérieur, qui, parce qu'il a une gestuelle civilisée (voir l'impact de la manière dont il fait remonter ses lunettes) ne peut être en réalité qu'un membre de la Fraternité, groupe subversif mené par Goldstein, l'ennemi public numéro un.

14 Ainsi, Orwell reprend la problématique de Benjamin : il fait vivre cette idée conceptuelle de la possibilité pour l'être de se réapproprier les expériences de liberté (différence) venues du passé et de pouvoir les saisir au présent. La particularité est que le régime totalitaire dans lequel se trouve enfermé Winston oblige à placer cette récupération des expériences de liberté du passé dans la sphère de l'intime et dans les replis les plus cachés de la subjectivité, car il n'y a pas d'espace de rencontre, d'échanges avec les autres qui soient permis par le Parti. 1984 est bien élaboré par Orwell dans ce double mouvement. D'un côté, le régime qui élimine le passé de la société pour asseoir une cohérence sociale sur l'absence de conflit ou de contradiction, de l'autre, le cheminement tâtonnant de Winston qui reconstruit une mémoire, une pensée et des liens: un retour sur soi qui conduit à la rupture. En conséquence, il y a dans cette exploration de sa propre subjectivité par le sujet une insistance sur la fertilité de cet espace de la subjectivité et de l'expérience intime pour créer de la politique, le désir de liberté, et d'émancipation. Orwell souligne le rapport étroit qu'il y a entre une construction dite interne et des relations se trouvant appartenir au domaine de l'externe. Entre les deux, il y a une perméabilité quotidienne. Le corps est une médiation indispensable entre ces deux termes, puisque c'est lui qui est le dépositaire des souvenirs et des expériences de Winston. Il a ainsi une place essentielle dans l'élaboration de l'autonomie individuelle, car il prend part à l'expérience de la différence si elle a lieu. Le corps de l'individu est à la fois une interface et un contenant, susceptible de devenir un premier espace de résistance. La révolte peut alors se trouver liée à des enjeux corporels. Par exemple, la sensualité opprimée de Julia l'entraîne à partager les idées de Winston. Le langage aussi sort de 
l'abstraction pour s'enraciner dans le charnel, constituant ainsi un autre espace de résistance à la novlangue. De plus, il est indispensable de souligner l'écart entre Winston et son amie Julia. Ils ont une différence d'âge qui fait que lui est né avant la révolution et elle non; et aussi une différence d'expérience : la relation affective de Winston avec sa mère, alors que Julia ne se souvient pas de ses parents, mais seulement de son grand-père. Tout cela fait que leurs attitudes sont totalement différentes. Alors que Winston se dégage mentalement de la fiction du Parti, elle ne s'en soucie pas, notamment dans les passages où elle s'endort lorsqu'il lui parle de la réécriture perpétuelle de l'histoire, ou encore lorsqu'il évoque le fait que la guerre change d'ennemi sans que Julia y accorde la moindre attention. L'important pour Julia est d'avoir le plus de liberté possible à l'intérieur du système totalitaire, et cela le plus longtemps possible. En réalité, elle s'accommode avec le système, sans penser qu'il pourrait être autre. Elle a choisi la transgression et non la rupture, comme Winston; et cet écart est manifeste tout au long de l'ouvrage, jusque dans la manière dont ils se dénoncent mutuellement. Elle est un « cas de manuel » pour O’Brien, alors que Winston, « esprit malade », résiste avant de céder devant l'horreur de sa peur, celle devant les rats ${ }^{12}$.

15 Si l'amour maternel que Winston a reçu est fondateur, il est aussi à l'origine de sa rupture. Il est sans le savoir le dépositaire d'un bien précieux qui l'entraîne à penser qu'un autre moyen de mise en relation entre les individus est possible. Son expérience affective enfantine lui permet de penser que des liens entre les individus peuvent exister. $\mathrm{Au}$ contraire, la servitude est sans doute plus grande là où il n'y a pas eu de liens aimants (la petite fille des voisins qui finit par dénoncer son propre père). D'ailleurs, il est intéressant à noter que lorsque Winston sort de son isolement, aime Julia, il cesse en même temps d'être malade : l'ulcère variqueux qu'il a sur le cou de pied cicatrise. Encore une fois, il est montré dans le récit combien le corps de l'individu a une place essentielle dans le rapport à autrui. Le corps de Winston, sa douleur au pied manifestent d'une douleur plus profonde - celle d'une absence de liens avec autrui. De même, l'article de Claude Lefort nous a montré que c'est le corps de l'autre, jeté, placé entre la torture et l'individu, qui conduit à la véritable trahison, qui rompt le lien entre les individus. C'est aussi dans la relation à l'autre par l'amour et l'intimité que l'individu connaît non seulement la jouissance, mais aussi poursuit le cheminement vers un autre monde possible. Winston Smith connaît la révolte par le passage à l'expérience de la mise en lien avec autrui. Deux choses qui sont vitales pour l'individu, mais qui se répondent dans le désir d'un être ensemble autre.

Sur cette question de l'amour et de la sexualité, nous sommes confrontés à un mouvement contradictoire, entre le système et le protagoniste. Contradiction entre les règles de vie de cette société (où l'amour est interdit et l'abstinence prônée comme une vertu, la haine étant le sentiment sur lequel se fait le partage entre les êtres), et la démarche du personnage qui cherche à reconstruire ce qui a été détruit par le Parti. Il s'agit de la possibilité d'être en lien qui est à l'origine de la possibilité d'un espace politique. Winston cherche à retrouver un dialogue à soi par l'usage du journal intime, mais il renoue aussi dans l'expérience amoureuse avec le dialogue à autrui. Sa quête et sa révolte se construisent en se nourrissant de ces expériences de vérité et d'hétéronomie. Faisant l'expérience de l'amour, il fait ainsi l'expérience de la différence et du conflit. Les possibilités d'expérimenter l'hétéronomie et le désaccord, sont au cœur de l'expérience politique et démocratique. 
17 L'attention portée ici sur les souvenirs et les rêves de Winston Smith nous permet de souligner que l'expérience de vie de l'individu est ce qui lui donne toute sa profondeur. Une profondeur qui est à l'origine des ressources permettant d'affronter et de rompre avec les plus puissantes fictions : celle des régimes qui sortent l'individu de la politique.

\section{Langage et expériences de la liberté} novlangue est une langue fabriquée de toutes pièces par les membres du Parti et cela, cas unique, en enlevant des mots. Imaginons, comme le Parti le projette, le moment où seule existera la novlangue. Les individus nés à ce moment-là n'auront rien de commun dans leur manière de penser avec ceux qui auront toujours parlé l'anglais et la novlangue.

Winston fait partie du second groupe, il possède un langage riche, dense, dans lequel il peut trouver des « contenants » pour ses sentiments. Julia, elle, emploie dans l'intimité un langage grossier. Winston est étonné et ravi de l'entendre jurer. Il y a là une satisfaction des deux personnages à entendre ce langage, à être "sales ", car c'est une forme de résistance pour eux à la doctrine du Parti : non seulement ils font l'amour, mais ils ont aussi une parole impure. En effet, les gros mots sont contraires à l'ordre ; en les utilisant, Julia transgresse l'ordre établi et, ce faisant, lève une censure. En parlant d'une manière interdite, elle va sauvegarder un terrain de liberté de l'être. Il y a comme une réaction charnelle de rejet du système chez Julia. Dans ce langage, elle retrouve une part d'ellemême, qui est bannie par le Parti. Ce langage est la marque de son identité, elle n'est pas une jeune militante à la ceinture rouge (évoquant la chasteté) mais bien une jeune femme sensuelle. Le langage ordurier lui permet donc de faire un travail de réappropriation de sa propre identité. De plus, le fait de lever une interdiction peut amener à en lever d'autres, à créer une dynamique. Winston, accompagné de Julia, va se décider à trouver O’Brien afin de faire partie de la Fraternité.

Winston se répète inlassablement que ce qui est n'a pas toujours été, et qu'il y a d'autres moyens de vie que ceux qui sont imposés par le Parti. Il se demande aussi s'il est le seul à avoir une mémoire. Le leitmotiv de Winston est profondément enraciné en lui, parce qu'il a un souvenir auquel il tient, celui d'avoir eu une mère aimante. Winston s'oppose, viscéralement, à la technique de la double pensée que tous emploient pour rester en harmonie avec le Parti ; car cette technique de la double pensée mise en place pour gommer les contradictions internes des individus doit en même temps effacer les souvenirs.

21 Cette technique de la double pensée est l'outil qui permet au Parti de compléter son action sur la parole, mais même cette technique d'autocensure a des limites ; l'individu le plus endoctriné et le plus bête ne peut le satisfaire ${ }^{13}$. La volonté du Parti décharne la langue, accompagne son désir de ne laisser aux êtres que les os du langage afin d'en faire des squelettes, des êtres sans épaisseur, sans profondeur, et sans pensée. L'appauvrissement du langage et le projet de le faire adhérer à la réalité la plus primaire détruisent la possibilité et la richesse du langage. La suppression de l'usage de la métaphore, du double sens, témoignent de ce désir de construire un langage sans obscurité, sans jeux, sans imagination. Océania veut construire un langage auquel «collent " les êtres, ce qui indique une idéologie qui veut accéder à la subjectivité des individus et qui veut surtout détruire toute possibilité d'intériorité, d'intimité des êtres, 
les laissant transparent au regard du Parti. Il y a là la visée de ne pas permettre l'évasion de l'être du monde et d'empêcher le repli sur soi et sur l'imagination. C'est le fantasme du Parti de réussir à créer des êtres qui ne pensent pas: ils ne peuvent avoir d'avis, ni surtout remettre en question la fiction dans laquelle ils vivent; ainsi ils se fondent en elle de façon parfaite.

\section{La fragilité du Grand Frère}

C'est là où nous touchons la plus grande faille du système totalitaire, son impossibilité à être d'un contrôle total. En effet, il ne peut réussir à arrêter l'accumulation d'expériences par les individus qu'il oppresse. Il ne peut réussir à écarter l'expérience de la vie des hommes. Cet acharnement à faire du vide non seulement autour mais à l'intérieur des êtres ne peut arrêter la production des expériences, aussi minimales soient-elles. Les sens des individus restent toujours en éveil dans le monde, même en plein désert, même dans la plus grande désolation.

23 La soumission peut alors connaître chez l'individu une limite, qui est dans l'identité même de l'individu et qui se constitue à partir de son expérience de la différence. C'est elle qui participe à la constitution de l'identité autonome de l'individu, et qui peut contredire la fiction du régime totalitaire. Chez Winston Smith, le rapport à sa mère lui permet de pouvoir contredire le régime de Big Brother en se rendant compte du décalage entre son vécu et les valeurs véhiculées par la propagande du régime. Par exemple, à travers les trois slogans du régime, «la guerre c'est la paix ; la liberté c'est l'esclavage ; l'ignorance c'est la force ${ }^{14} »$. Ce rapport, qui est une expérience de la différence, va faire éclater la contradiction entre l'individu et le régime totalitaire. L'individu peut alors porter un regard sur la fiction du régime et être en situation de lui donner un sens, de l'interpréter. La révolte est d'abord une distanciation entre l'individu et la fiction du régime totalitaire. Cette distanciation nécessite un pouvoir d'interprétation de l'individu qui est lié à sa capacité de ressentir les expériences qu'il réalise.

\section{L'essai et le roman}

Une fois que la rupture est pensée, Winston se jette dans « la gueule du loup » et s'adresse à 0 'Brien. Winston cherche un groupe, un espace, où sa pensée et ses expériences peuvent s'inscrire dans une action. Il se tourne alors vers la seule personne qui lui apparait comme pouvant être membre du mouvement dissident. Cela montre la nécessité pour l'individu de trouver un espace où il puisse faire l'expérience de sa révolte. Un espace collectif.

Dans le roman d'Orwell c'est justement là que se tient la césure narrative. En effet, dans 1984, Winston Smith est brisé à partir du moment où il décide de se diriger vers un acteur supposé de la résistance à Big Brother. Il se dirige vers O'Brien, car il a une confiance aveugle dans ce qu'il croit lire en lui : un être qui fait vivre des expériences venues du passé, et des expériences que lui, Winston, reconnait comme étant expression de la liberté. Le personnage en trompe-l'oeil d'o'Brien est ainsi le leurre dans lequel tombe Winston, croyant trouver la brèche vers l'organisation de lutte contre l'oppression totalitaire. En réalité, Winston s'adresse à son futur bourreau et fidèle serviteur du régime d'Océania. 
Le livre se termine apparemment par la réhabilitation du personnage après avoir été torturé et finalement remis dans le droit chemin. Il y aurait alors une fermeture du récit sur l'échec de cette révolte individuelle, car elle ne peut trouver de forme collective de lutte et de résistance. Orwell laisserait alors le lecteur désemparé face à l'insurmontable de la situation. Cependant, le roman ne se termine pas avec la fin du récit, mais s'ouvre sur un essai sur la novlangue, une analyse qui permet de saisir ce qu'était la novlangue. Ainsi, l'auteur parvient à réaliser un mélange des genres. Il introduit la forme fragmentaire de l'essai pour indiquer un élément essentiel du roman. C'est là l'élément essentiel : ce qu'était la novlangue. Ainsi, l'essai nous laisse pressentir par l'usage des temps que fait l'auteur que le régime et la novlangue ont échoué et ne se sont ni réalisés ni maintenus. Il y a ainsi une césure dans le récit et une ouverture : le régime totalitaire a été mis à bas. Seulement, Orwell ne nous indique pas le chemin à parcourir pour faire tomber cette oppression. Il y a ainsi chez le romancier une grande finesse, car non seulement il y a une critique de l'oppression qui se maintient en indiquant l'ampleur de la force du régime et en mettant l'accent sur le destin tragique de Winston Smith, mais il y a aussi et dans le même temps l'inscription d'une ouverture : ce régime a été mis à bas et son projet détruit. Orwell réalise ce mouvement dans la mise en place d'une solution d'écriture superbe, dialectique et benjaminienne, par une inscription des temps que choisit l'auteur ${ }^{15}$. Par l'essai sur la novlangue, il y a un retour par le passé - de l'écriture et de la lecture - rendant pensable un futur du temps à venir, celui où l'oppression sera détruite. La lecture de 1984 nous permet de faire surgir le futur - un futur qu'on pourrait imaginer utopique. Orwell nous laisse ainsi vivre en même temps l'expérience de la soumission et celle de la révolte, mais aussi l'expérience de la liberté inscrite à tout jamais dans 1984. Le titre lui-même en est l'écho, car écrit en 1948, il se présentait tout à la fois comme un avenir possible, mais aussi un passé de la lecture et des temps dès que l'année 1984 serait passée. Ainsi, 1984 devient un moyen de transmettre l'expérience de la liberté venue du passé. À notre tour de nous en souvenir.

\section{NOTES}

1. George Orwell, 1984, Gallimard, Paris, 1976.

2. Karl Marx, Le 18 Brumaire de Louis Bonaparte, Mille et une nuits, 1997, p. 13 : «La tradition de toutes les générations mortes pèse d'un poids très lourd sur le cerveau des vivants ». Nous usons d'une version revue par nos soins.

3. Voir le rapport de l'Inserm qui établit un lien entre « les facteurs à risque » chez les petits et les « trajectoires délinquantes ", Le Canard enchaîné, nº 4454, mercredi 8 mars, 2006.

4. Claude Lefort, « Le corps interposé », in Passé Présent, Ramsay, Paris, 1984.

5. Cet élément du journal intime est déjà présent dans l'ouvrage d'anticipation We de Yevgeny Zamyatin, écrit en 1921. Voir Yevgeny Zamyatin, We, Penguin Books, GB, 1993.

6. Hannah Arendt, Le système totalitaire, les origines du totalitarisme, Seuil, Paris, 1972.

7. Ce terme de distopique est repris à Juan Manuel Santiago, jeune auteur espagnol et spécialiste de la science-fiction. 
8. Cette année l'Académie française a introduit le terme de novlangue dans la langue française. Il s'agit là, non seulement d'une véritable reconnaissance de la richesse créative d'Orwell, mais aussi d'une mesure de l'impact du roman sur les individus et la société.

9. Friedrich Nietzsche, La Généalogie de la morale, Folio essais, Gallimard, 1971, II, § 13-16.

10. Sigmund Freud, Malaise dans la culture, Quadrige/Presses Universitaires de France, Paris 1995, p. $11:$ « [...] rien de ce qui s'est une fois produit n'a disparu [...]»

11. Il faut tenir compte du contexte historique dans lequel l'auteur réalise ce roman. Il voit l'avènement $\mathrm{du}$ fascisme espagnol, du nazisme et de l'Union soviétique. Il vit et combat le surgissement de ces régimes, et il sera l'un des premiers à les penser et à en cerner l'étendue.

12. Claude Lefort, op. cit., p. 15.

13. Cf. le voisin, parfait membre du Parti, qui se retrouve à crier à bas Big Brother quand il dort, pp. 330-332).

14. Orwell, op. cit., p. 15.

15. J'aborde cette question de l'essai, pour Adorno et Benjamin, dans mon article « La Théorie critique : une pensée qui fait désordre ", in Variations, La Théorie critique, Héritages hérétiques, automne 2005.

\section{AUTEUR}

\section{LUCIA SAGRADINI}

Attachée de recherche et d'enseignement en sociologie politique à l'Université Paris 7 